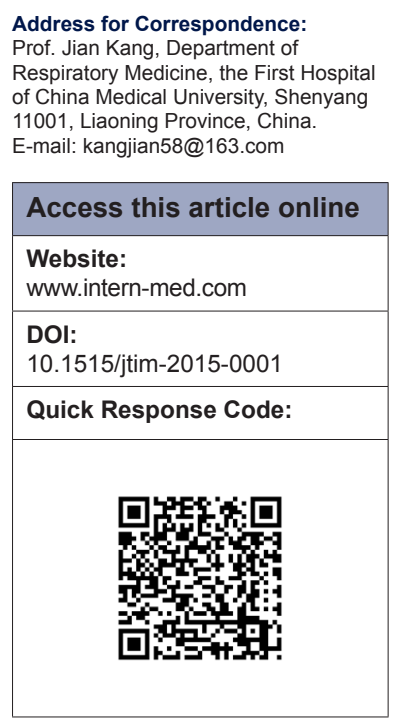

\section{More attention to comprehensive assessment and individualized therapy of chronic obstructive pulmonary disease}

\author{
Gang Hou, Jian Kang \\ Department of Respiratory Medicine, First Hospital of China Medical University, Shenyang 11001, China
}

\section{INTRODUCTION}

Chronic obstructive pulmonary disease (COPD) is a largely preventable and treatable disease characterized by airflow limitation. The airflow limitation is usually progressive and associated with a chronic inflammatory response of the airways and lungs to noxious particles or gases ${ }^{[1]}$. Chronic obstructive pulmonary disease is heterogeneous and assumes different phenotypes. COPD patients with different phenotypes have different clinical features and characteristics, giving rise to difference in prognosis and responses to treatment. Along with the development of precision medicine, individualized therapy or specific treatment has received increasing attention and wide application. To focus on and implement individualized treatment is the core of the diagnosis and management of chronic obstructive pulmonary disease. Systematic assessment of the disease features and severity is the basis for individualized treatment.

\section{WHAT ARE PHENOTYPES AND CLASSIFICATION OF COPD?}

A phenotype is the composite of an organism's observable characteristics or traits, such as its morphology, development, biochemical or physiological properties, phenology, behavior, and products of behavior. It is influenced by heredity and external environment. Recently, phenotypes have been widely used in classification of chronic obstructive pulmonary disease, which aims to discover the unique prognosis or management of subgroups of COPD patients and to guide individual treatment ${ }^{[2]}$.

Currently, commonly used are following three methods of phenotyping in clinical practice: the clinical phenotypes, respiratory phenotype and imaging phenotype.

\section{Clinical phenotypes}

Chronic bronchitic phenotype, emphysematic phenotype, and chronic bronchititis-emohysema overlap phenotype: Clinically, some patients with COPD present recurrent productive cough, which is due to prominent inflammatory changes of the airways; some COPD patients, in the absence of productive cough, are characterized by progressive exertioninduced shortness of breath, which is due to excessive ventilation, decreased elascity and lowered lung compliance and diaphoretic activity. However, some COPD patients have both the above clinical manifestations. The above three are respectively classified as the chronic bronchitic phenotype, emphysematic phenotype, and chronic bronchitis-emohysema overlap phenotype. It should be noted that the diagnosis of emphysema needs to be confirmed by imaging studies. For this reason, clinical manifestations alone are not sufficient for accurate phenotyping. High resolution computed tomography is essential to its diagnosis.

Frequent exacerbations: Frequent exacerbations in COPD patients would prompt its progression, giving rise to increased mortality and a poor prognosis. Therefore, it is recommended that COPD patients with a history of frequent exacerbations (defined as exacerbation 
more than twice per year) are considered as a unique clinical phenotype, named frequent acute exacerbation phenotype. Such patients should be educated on how to recognize acute exacerbations. Meanwhile, a close follow-up helps to obtain information about the frequency of exacerbation and helps therefore for further classification of phenotypes. In addition, since there are various factors which may give rise to acute exacerbations, careful assessment of risk factors for exacerbation as well as specific interventions, help to alleviate acute exacerbations and improve quality of life and prognosis.

Asthma-COPD overlap syndrome (ACOS): Currently, it is held that COPD and asthma are two different kinds of chronic airway disease, but in reality, COPD patients may present with airway hyper-responsiveness and recurrent wheezing. Such patients have the characteristics of both asthma and COPD. For this reason, ACOS may be a special phenotype of COPD. ACOS patients are prone to dyspnea, wheezing and are at a greater risk for acute exacerbations, with lowered quality of life and reduced tolerance to exercise ${ }^{[5]}$. Currently, no standard diagnostic criteria for ACOS have been established. Moreover, there has been no evidence for therapeutic efficacy and clinical outcomes, which awaits further studies. However, inhaled corticosteroids in combination with long-acting $\beta 2$-agonists and/or long-acting inhaled anticholinergic medication, rather than long-acting $\beta 2$-agonists alone, are recommended.

COPD and obstructive sleep apnea syndrome phenotype: Some COPD patients may also develop obstructive sleep apnea syndrome. As a result, with characteristics of both diseases, they develop an overlap syndrome. As compared with patients with simple obstructive sleep apnea syndrome, the respiratory center of patients with overlap syndrome may have lowered sensitivity to nocturnal hypoxia and hypercapnia stimulation. Consequently, such patients are more likely to develop sleep disorders, which prompts more severe nocturnal hypoxia and hypercapnia, creating a vicious cycle and aggravating chronic obstructive pulmonary disease. Due to therapeutic difference in the management of COPD and obstructive sleep apnea syndrome, once a missed diagnosis of the overlap syndrome is made and no appropriate intervention is offered, its prognosis would be affected. Therefore, for COPD patients who are at high risk for obstructive sleep apnea, polysomnography should be performed to confirm the presence of the overlap syndrome.

\section{Respiratory phenotype}

Research found that the decline rate in forced expiratory volume in 1 second (FEV1) can predict frequency of exacerbations, time to the first acute exacerbation, quality of life and mortality ${ }^{[6]}$, and the rapid decline in FEV1 was recommended as a phenotype of COPD. It should be noted that the rate of annual decline in FEV1 varies greatly among individuals, which is higher in patients with mild-to-moderate than those with very severe COPD. This finding highlights the importance of early management for chronic obstructive pulmonary disease ${ }^{[7]}$. Therefore, to promote and popularize pulmonary function tests in primary health care units, to monitor pulmonary changes in COPD patients, and to calculate the annual decline in FEV1, may be beneficial to treatment and judgment of prognosis and can slow the rapid decline in lung function.

\section{Imaging phenotype}

HRCT quantitative data analysis can better assess airway conditions as well as characteristics and severity of emphysema. According to the findings of HRCT, Kitaquchi et al classified COPD into the emphysema phenotype, bronchial wall thickening phenotype, and emphysema and bronchial wall thickening phenotype ${ }^{[8]}$. Research findings revealed that severity of emphysema in COPD patients was positively correlated with annual packs of cigarettes, and negatively associated with BMI, DLco and FEV1/FVC. Moreover, the presence of bronchial wall thickening was significantly associated with responsiveness to treatment with inhaled corticosteroid as well as eosinophilia. HRCT quantitative data analysis can not only be used for phenotypes, but also for heterogeneity of emphysema and interlobar fissure integrity analysis. It also helps develop individualized therapies, for example, bronchoscopic lung volume reduction surgery.

\section{COMPREHENSIVE ASSESSMENT}

The goal of comprehensive assessment of COPD is to determine the severity and impact of the disease on quality of life as well as the risk for the long-term future events (such as acute exacerbation, hospitalization or death), so as to guide individualized treatment programs. Mainly the following four aspects are assessed, including current symptoms, severity of lung function, risk for acute exacerbation and presence of complications.

\section{COPD assessment test}

As to the assessment of symptoms, clinically, COPD assessment test (CAT) and COPD control questionnaire are used for systemic assessment. CAT's correlation with the COPD-specific version of the St George's Respiratory Questionnaire is good, therefore they are simple and reliable $^{[0]}$. The critical point of CAT is 10 points. CAT $<10$ points indicates less symptoms, whereas CAT $>10$ points indicates more symptoms. Due to a lack of critical points, 
the commonly-used modified Medical Research Council (mMRC) dyspnea scores fail to reveal the comprehensive status of the patient. For this reason, (mMRC) score is not recommended to be used alone for assessment. However, $m M R C \geq 2$ still serves as a cut-point for the differentiation between "less dyspnea" and "more dyspnea".

\section{FEV1}

The severity of lung function is still determined based on the proportion of FEV1 percent predicted after the use of bronchodilators. The critical point for grading lung function is used not because it is evidence-based, but just because it is simple. Lung function grading is poorly associated with symptoms and quality of life. For this reason, COPD assessment should not be over-reliant on lung function grading. Instead, a comprehensive assessment is required.

\section{Risk assessment of acute exacerbation in COPD}

Acute exacerbation of COPD is an acute event in the progression of COPD, mainly manifesting as worse respiratory symptoms which are beyond the normal variation and need medication. Assessment of COPD exacerbation is based on symptoms and therapeutic effects whereas risk assessment of COPD exacerbation is mainly based on the history of acute exacerbation of COPD and severity of lung function, as studies have shown that acute exacerbation (exacerbations $\geq 2$ per year) is a best indicator for acute exacerbation. Moreover, people with GOLD stageIII and GOLD stage IV are at greater risk for acute exacerbation ${ }^{[10]}$. As COPD may prompt a rapid decline in lung function, quality of life, and an increased risk for death, risk assessment of acute exacerbation can also be considered as a risk assessment of long-term adverse prognosis.

\section{Assessment of co-morbidity}

Co-morbidity may exert impact on the severity of COPD. There are three risk factors for co-morbidities: Presence of smoking. Smoking is a risk factor for many diseases. Smokers with COPD are more likely to develop lung cancer and cardiovascular diseases; age-related complications; extra-pulmonary manifestations of chronic obstructive pulmonary disease, including weight loss, malnutrition, wasting muscle, etc. Given co-morbidities were independent risk factors that affect hospitalization and death in COPD patients, in addition to the routine treatment of COPD, appropriate attention should also be given to the assessment and treatment of its co-morbid conditions.

\section{DIFFERENCE IN THERAPEUTIC RESPONSE AMONG DIFFERENT}

\section{PHENOTYPES}

Due to difference in mechanism and characteristics of disease, patients with different phenotypes may exhibit different responses even to the same therapy. Current research mainly focused on difference in responses between chronic bronchitis phenotype and emphysema phenotype. Studies have shown that, as compared with people with chronic bronchitic phenotype and overlap phenotype, people with emphysema phenotype did not respond well to long-acting beta-agonist or inhaled corticosteroids ${ }^{[1]]}$. However, a study in china regarding treatment of COPD with phosphodiesterase 4 inhibitors showed that Roflumilast exhibited marked improvement in prebronchodilator FEV1 for people with chronic bronchitis ${ }^{[12]}$. Rennard et al showed Roflumilast can not reduce acute exacerbation for patients with emphysema phenotype, whereas it can significantly reduce acute exacerbations for patients with chronic bronchitic phenotype $e^{[13]}$. Thus Roflumilast may be more suitable for patients with chronic bronchitis phenotype. Currently, studies on frequent exacerbation phenotype show that inhaled corticosteroid is more suitable for patients with frequent exacerbation phenotype, which can reduce the number of exacerbations, and improve symptoms and quality of life. Inhaled coticosteroids are thus recommended for COPD patients with subgroups $C$ and $\mathrm{D}^{[14]}$. In addition, the study regarding the treatment of COPD with macrolides showed that azithromycin maintenance therapy can significantly reduce the frequency of acute exacerbations in patients with exacerbation phenotype, and delay the time to the first acute exacerbation ${ }^{[15]}$.

\section{CONCLUSION}

In summary, chronic obstructive pulmonary disease has heterogeneity and assumes several phenotypes with different clinical characteristics, treatment responses and clinical outcomes. Due to difference in methods of classification, there are many phenotypes present even in one single patient. Therefore, the systemic assessment of disease conditions, detailed disease characteristics and stratified classification and individualized treatment according to the severity of the disease, indications and the patient's response to drug therapy are basic strategies for COPD management, which should be valued by clinical physicians.

\section{REFERENCES}

1. Vestbo J, Hurd SS, Agustí AG, Jones PW, Vogelmeier C, Anzueto A, et al. Global strategy for the diagnosis, management, and prevention of chronic obstructive pulmonary disease: GOLD executive summary. Am J Respir Crit Care Med 2013; 187:347-65.

2. Burgel PR, Paillasseur JL, Tillie-Leblond I, Tillie-Leblond I, Chanez P, 
Escamilla R, et al. Clinical COPD phenotypes: a novel approach using principal component and cluster analyses. Eur Respir J 2010;36: 531-9.

3. Koblížek V, Chlumský J, Zindr V, Neumannová K, Zatloukal J, Sedlák

$\mathrm{V}$, et al. Chronic obstructive pulmonary disease in the light of new guidelines-brief summary of phenotypically oriented guidelines for non pulmonary physicians. Vnitr Lek 2013; 59: 505-14.

4. Wedzicha JA, Brill SE, Allinson JP, Donaldson GC. Mechanisms and impact of the frequent exacerbator phenotype in chronic obstructive pulmonary disease. BMC Med 2013; 14: 181

5. Miravitlles M, Soriano JB, Ancochea J, Muñoz L, Duran-Tauleria E, Sánchez G, et al. Characterisation of the overlap COPD-asthma phenotype: Focus on physical activity and health status, Respir Med. 2013; 107: 1053-60.

6. Kesten S, Celli B, Decramer M, Liu D, Tashkin D. Adverse health consequences in COPD patients with rapid decline in FEV1 evidence from the UPLIFT trial. Respir Res 2011; 12: 129-31.

7. Nishimura M, Makita H, Nagai K, Konno S, Nasuhara Y, Hasegawa $\mathrm{M}$, et al. Annual change in pulmonary function and clinical phenotype in chronic obstructive pulmonary disease. Am J Respir Crit Care Med 2012; 185: 44-52.

8. Kitaquchi Y, Fujimoto K, Kubo K, Honda T. Characteristics of COPD phenotypes classified according to the findings of HRCT. Respir Med 2006; 100: 1742-52.
9. Jones PW, Harding G, Berry P, Wiklund I, Chen WH, Kline Leidy N Development and first validation of the COPD Assessment Test. Eur Respir J 2009; 34: 648-54

10. Hurst JR, Vestbo J, Anzueto A, Locantore N, Müllerova H, Tal-Singer $\mathrm{R}$, et al. Susceptibility to exacerbation in chronic obstructive pulmonary disease. N Engl J Med 2010; 363: 1128-38.

11. Lee JH, Lee YK, Kim EK, Kim TH, Huh JW, Kim WJ, et al. Responses to inhaled long-acting beta-agonist and corticosteroid according to COPD subtype. Respir Med 2010; 104: 542-9.

12. Zheng JP, Yang JH, Zhou XD, Zhao L, Hui F, Wang H, et al. Roflumilast for the treatment of COPD in an Asian population: A randomized, double-blind, parallel-group study. Chest 2014; 145: 44-52.

13. Rennard SI, Calverley PMA, Geohring UM, Bredenbröker D, Martinez FJ. Reduction of exacerbation by the PDE4 inhibitor roflumilast - the importance of defining different subsets of patients with COPD. Respir Res 2011; 12: 18-27.

14. Nannini LJ, Lasserson TJ, Poole P. Combined corticosteroid and long-acting beta(2)-agonist in one inhaler versus long-acting beta(2)agonists for chronic obstructive pulmonary disease. Cochrane Database Syst Rev 2012; 9: CD006829.

15. Albert RK, Connett J, Bailey WC, Casaburi R, Cooper JA Jr, Criner GJ, et al. Azithromycin for prevention of exacerbations of COPD. N Engl J Med 2011; 365: 689-98.

How to cite this article: Hou G, Kang J. More attention to comprehensive assessment and individualized therapy of chronic obstructive pulmonary disease. J Transl Intern Med 2015;3:39-42.

Source of Support: Nil, Conflict of Interest: None declared. 